\title{
Analysis of the prognosis of patients with testicular seminoma
}

\author{
WEI DONG ${ }^{1}$, WANG GANG ${ }^{1}$, MIAOMIAO LIU ${ }^{2}$ and HONGZHEN ZHANG ${ }^{2}$ \\ ${ }^{1}$ Department of Urology; ${ }^{2}$ Department of Fifth Oncology, Hebei People's Hospital, Shijiazhuang, Hebei 050051, P.R. China
}

Received November 23, 2015; Accepted December 30, 2015

DOI: $10.3892 / 01.2015 .4065$

\begin{abstract}
Testicular seminoma is a common malignancy, accounting for $35-50 \%$ of testicular tumors. Comprehensive therapies lead to good curative efficacy. However, the factors that affect prognosis remain to be elucidated. The aim of the present study was to analyze the 3-, 5- and 10-year survival rate of patients with testicular seminoma as well as the associated factors of prognosis. The clinical data from 58 patients diagnosed with testicular seminoma were collected between January 1999 and January 2014. The survival rate for this group was evaluated using the Kaplan-Meier method. Associated factors of prognosis were analyzed using the log-rank test. The results showed that approximately $62.1 \%$ of the cases were in the 30- to 50-year age group. From this age group, $94.8 \%$ of patients survived for 3 years, $86.2 \%$ for 5 years and $70.7 \%$ for 10 years. A significant difference was identified for the different clinical stages, pathological types and postoperative treatment in the 3-, 5- and 10-year survival rates $(\mathrm{P}<0.05)$. In conclusion, the clinical stages, pathological types and postoperative treatments significantly affect the prognosis of testicular seminoma. Selection of an appropriate method of treatment including the clinical stages and histological types, is the key element in testicular seminoma therapy.
\end{abstract}

\section{Introduction}

Testicular seminoma accounts for $40 \%$ of primary testicular neoplasms, with $70-85 \%$ of patients presenting with disease confined to the testis (Stage I), while 15-20\% present with infradiaphragmatic lymphadenopathy (Stage II) (1). Prognostic factors of testicular seminoma include age and pathology of the primary tumor: small vessel invasion, tumor size, and invasion of rete testis (1). In the current study, a retrospective analysis was conducted on the 3-, 5- and 10-year survival rates of patients with testicular seminoma in 58 different cases.

Correspondence to: Dr Hongzhen Zhang, Department of Fifth Oncology, Hebei People's Hospital, 348 Heping West Street, Shijiazhuang, Hebei 050051, P.R. China

E-mail: ai454w@163.com

Key words: testicular seminoma, survival rate, prognosis
The aim of the study was to determine factors that potentially affect prognosis in order to provide a guideline for clinical treatment and follow ups.

\section{Materials and methods}

Clinical materials. A total of 58 patients diagnosed with testicular seminoma at the People's Hospital of Hebei between January 1999 and January 2014 were enrolled in the current study. The age range of the patients was 23-75 years (average age, 36 years). The tumors in 35 cases were located on the right side and the remaining 23 cases were located on the left side. Pathological type was identified in 29 cases diagnosed with typical testicular seminoma, 13 cases with testicular seminoma combined embryonal carcinoma, and 16 cases with testicular seminoma combined embryonal carcinoma as well as teratoma. Clinical stages were classified as: 17 cases in stage I, 32 cases in stage II, and 9 cases in stage III. Lactate dehydrogenase (LDH), human chorionic gonadotropin (HCG) and $\alpha$-fetoprotein (AFP) were measured prior to the treatment. It was found that 34 cases had high LDH (normal value: 109-245 IU/1), accounting for $58.6 \%$ of the cases; 26 or $44.8 \%$ of the cases had higher than normal HCG (normal value $<2.6 \mathrm{mIU} / \mathrm{ml}$ ); and 27 cases or $46.6 \%$ had high AFP (normal value $<7.0 \mathrm{ng} / \mathrm{ml}$ ). All 58 cases were treated with radical orchiectomy, with 35 cases undergoing post-operation radiotherapy, 12 cases postoperative chemotherapy, and 11 cases post-operation radiotherapy combined with chemotherapy.

Methods used. Patient clinical data, including age, tumor position, pathological type, clinical stage, AFP, HCG and LDH levels, as well as postoperative treatment methods were identified and classified (Table I). Subsequently, the patients' conditions during the follow-up period following surgery were recorded. The survival rate was also calculated and prognosis was analyzed.

Follow-ups. The follow-up process was performed by a variety of means, including primarily phone calls, clinical visits and reexaminations. Follow ups were mainly focused on patient postoperative treatment, survival state, and survival time. Survival time was calculated by months from the first time they underwent therapy until the last follow-up. Death events were defined as the end point. Patient survival or lost follow-up data were processed as censored data. Deadline for the follow ups was December 31, 2014. 
Statistical analysis. Statistical analysis was performed using SPSS 13.0 (SPSS, Inc., Chicago, IL, USA) statistical software. The Kaplan-Meier survival curve was used to analyze the survival rate and time. The log-rank single factor analysis was used to analyze and examine the differences between the subgroups. $\mathrm{P}<0.05$ was considered to indicate a statistically significant difference.

\section{Results}

Clinical characteristics of testicular seminoma

Association between testicular seminoma and age. Of the 58 patients, 36 were aged 30-50 years, accounting for $62.1 \%$ of all the cases. The incidence therefore was relatively high (Table II).

Association between testicular seminoma and testicular side. In 35 cases (60.3\%) tumors were located on the right side, and in 23 cases (39.7\%) tumors were located on the left side. The results showed the number of cases with right-side tumors was superior to that of cases with left-side tumors (Table III).

Association between testicular seminoma and pathological types. Of the 58 cases, 29 cases were confirmed with typical testicular seminoma, 13 cases with testicular seminoma combined with embryonal carcinoma and 16 cases with testicular seminoma combined with embryonal carcinoma and teratoma, accounting for 50.0, 22.4 and $27.6 \%$, respectively, of the testicular seminoma patients. The proportion of typical testicular seminoma identified was relatively higher (Table IV).

Clinical stage of testicular seminoma. Testicular seminoma was identified in the early stages. In the present study, 49 cases were in stages I and II, accounting for $84.5 \%$ (Table V).

Testicular seminoma treatment methods. Testicular seminoma is highly sensitive to radiotherapy and its recurrence rate following radiotherapy is extremely low. In the present study, 35 cases, accounting for $60.3 \%$ of cases, were treated with radiotherapy. The postoperative chemotherapy regimen administered was 3-cycle PEB regimen or 4-cycle EP regimen, and for patients in stage IIIC the 4-cycle PEB regimen was administered (Table VI).

\section{Kaplan-Meier survival rate calculation}

Analysis of the survival rate. Results of the survival rate analyses revealed that $94.8 \%$ of the patients survived for 3 years after orchiectomy while $86.2 \%$ survived for 5 years and $70.7 \%$ for 10 years after orchiectomy (Fig. 1).

Postoperative survival rate for different pathological types. The 3-year survival rate for patients diagnosed with typical testicular seminoma was $100 \%, 93.8 \%$ for cases with testicular seminoma combined with embryonal carcinoma, and $84.6 \%$ for those with testicular seminoma combined with embryonal carcinoma and teratoma. The 5-year survival rate for patients diagnosed with typical testicular seminoma was $93.1 \%, 87.5 \%$ for cases with testicular seminoma combined with embryonal carcinoma, and $69.2 \%$ for those with testicular seminoma combined with embryonal carcinoma and teratoma. The
Table I. Log-rank single-factor analysis.

\begin{tabular}{|c|c|c|c|}
\hline Clinical factor & Case & $\chi^{2}$ test & P-value \\
\hline Age, years & & 0.785 & 0.940 \\
\hline $20-29$ & 6 & & \\
\hline $30-39$ & 20 & & \\
\hline $40-49$ & 16 & & \\
\hline $50-59$ & 9 & & \\
\hline$>59$ & 7 & & \\
\hline Tumor side & & 0.906 & 0.341 \\
\hline Right & 35 & & \\
\hline Left & 23 & & \\
\hline Pathological side & & 9.070 & 0.011 \\
\hline $\begin{array}{l}\text { Typical testicular } \\
\text { seminoma }\end{array}$ & 29 & & \\
\hline $\begin{array}{l}\text { Testicular seminoma } \\
\text { combined embryonal } \\
\text { carcinoma }\end{array}$ & 13 & & \\
\hline $\begin{array}{l}\text { Testicular seminoma } \\
\text { combined embryonal } \\
\text { carcinoma as well as teratoma }\end{array}$ & 16 & & \\
\hline Clinical stage & & 7.162 & 0.028 \\
\hline I & 17 & & \\
\hline II & 32 & & \\
\hline III & 9 & & \\
\hline LDH value & & 5.285 & 0.022 \\
\hline Normal & 24 & & \\
\hline Increasing & 34 & & \\
\hline HCG value & & 3.677 & 0.055 \\
\hline Normal & 32 & & \\
\hline Increasing & 26 & & \\
\hline AFP value & & 4.783 & 0.029 \\
\hline Normal & 31 & & \\
\hline Increasing & 27 & & \\
\hline $\begin{array}{l}\text { Postoperative } \\
\text { treatment methods }\end{array}$ & & 6.362 & 0.042 \\
\hline $\begin{array}{l}\text { Postoperative } \\
\text { radiotherapy }\end{array}$ & 35 & & \\
\hline $\begin{array}{l}\text { Postoperative } \\
\text { chemotherapy }\end{array}$ & 12 & & \\
\hline $\begin{array}{l}\text { Postoperative } \\
\text { radiotherapy+chemotherapy }\end{array}$ & 11 & & \\
\hline
\end{tabular}

LDH, lactate dehydrogenase; HCG, human chorionic gonadotropin; AFP, $\alpha$-fetoprotein.

10-year survival rate for patients diagnosed with typical testicular seminoma was $86.2 \%, 68.8 \%$ for cases with testicular seminoma combined with embryonal carcinoma, and 38.5\% for those with testicular seminoma combined with embryonal carcinoma and teratoma (Fig. 2).

Postoperative survival rate of different clinical stages. The 3 -year survival rate for patients in different clinical stages of 
Table II. Association between the age and the occurrence of testicular seminoma.

\begin{tabular}{lcc}
\hline Age & Case & Proportion, \% \\
\hline $20-29$ & 6 & 10.3 \\
$30-39$ & 20 & 34.5 \\
$40-49$ & 16 & 27.6 \\
$50-59$ & 9 & 15.5 \\
$>59$ & 7 & 12.1 \\
Total & 58 & 100.0 \\
\hline
\end{tabular}

Table III. Association between testicular seminoma and testicle side.

\begin{tabular}{lcc}
\hline $\begin{array}{l}\text { Tumor side } \\
\%\end{array}$ & Case & Proportion, \\
\hline Left & 23 & 39.7 \\
Right & 35 & 60.3 \\
Total & 58 & 100.0 \\
\hline
\end{tabular}

Table IV. Association between testicular seminoma and pathological types.

\begin{tabular}{lcc}
\hline Pathological type & Case & Proportion, \% \\
\hline $\begin{array}{l}\text { Typical testicular } \\
\text { seminoma }\end{array}$ & 29 & 50.0 \\
$\begin{array}{l}\text { Testicular seminoma } \\
\text { combined embryonal } \\
\text { carcinoma }\end{array}$ & 13 & 22.4 \\
$\begin{array}{l}\text { Testicular seminoma } \\
\text { combined embryonal } \\
\text { carcinoma as well as } \\
\text { teratoma }\end{array}$ & 16 & \\
Total & & 27.6 \\
\hline
\end{tabular}

cancer was: $100 \%$ for stage I, $93.8 \%$ for stage II and $88.9 \%$ for stage III. The 5-year survival rate was 94.1, 87.5 and $66.7 \%$ for stages I, II and III, respectively. The 10-year survival rate for stage I patients was $88.2 \%$ and for stages II and III patients 71.9 and $33.3 \%$, respectively (Fig. 3).

Survival rate for the different treatment methods. The 3-year survival rate for postoperative radiotherapy, postoperative chemotherapy and postoperative radiotherapy combined with chemotherapy was analyzed in the present study. The results showed that all patients (100\%) survived for 3 years following treatment with postoperative radiotherapy. For patients who underwent postoperative chemotherapy an 83.3\% 3-year survival rate was observed. Ninety percent of our cases who were treated with postoperative radiotherapy combined with chemotherapy survived for 3 years following treatment.
Table V. Association between testicular seminoma and clinical stages.

\begin{tabular}{lcc}
\hline Clinical stage & Case & Proportion, \% \\
\hline I & 17 & 29.3 \\
II & 32 & 55.2 \\
III & 9 & 15.5 \\
Total & 58 & 100.0 \\
\hline
\end{tabular}

Table VI. Association between testicular seminoma and treatment methods.

\begin{tabular}{lcc}
\hline Treatment methods & Case & Proportion, \% \\
\hline Postoperative radiotherapy & 35 & 60.3 \\
Postoperative chemotherapy & 12 & 20.7 \\
$\begin{array}{l}\text { Postoperative radiotherapy } \\
\text { and chemotherapy }\end{array}$ & 11 & 19.0 \\
Total & 58 & 100.0 \\
\hline
\end{tabular}

The 5-year survival rate was analyzed for the abovementioned set of treatments. The results showed that $91.4 \%$ of cases survived for 5 years subsequent to their treatment by postoperative radiotherapy. For patients who underwent postoperative chemotherapy a $75.0 \%$ 5-year survival rate was observed and $81.8 \%$ for those treated with postoperative radiotherapy combined with chemotherapy (Fig. 4).

Results for the log-rank single factor analysis. The log-rank single factor analysis results indicated that the clinical stage, pathological type and postoperative treatment methods were statistically significant with regard to the prognosis of the patients with testicular seminoma $(\mathrm{P}<0.05)$. Preoperative LDH and AFP levels also affected their prognosis $(\mathrm{P}<0.05)$ (Table I).

Results of the multiple factor analysis. The results from the multiple factor analysis indicated that the influence of clinical stage and postoperative treatment methods was statistically significant on patient prognosis $(\mathrm{P}<0.05)$.

\section{Discussion}

General. Testicular tumor is a relatively rare cancer, ranked 6th of the top common male tumors in the United States (2). Testicular seminoma is the most common type of all testicular tumors. Most testicular seminoma are identified in the early stages and patients have a good recovery under the assistance of normative chemotherapy and radiotherapy following orchiectomy. Therefore, testicular tumor is a typical example of the treatable cancer types (3). The results of the present study showed that the 3-, 5- and 10-year survival rates for testicular seminoma patients were $94.8,86.2$ and $70.7 \%$, respectively, indicating a relatively high survival rate following orchiectomy. 


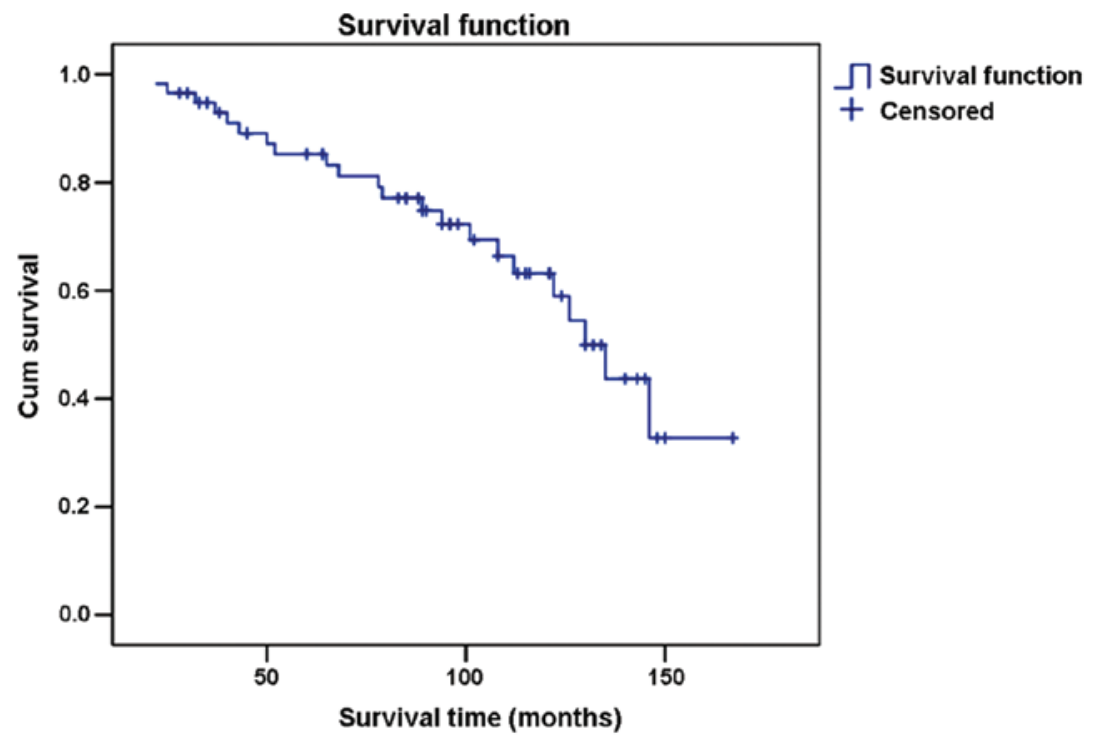

Figure 1. Survival curve for 58 testicular seminoma patients after orchiectomy.

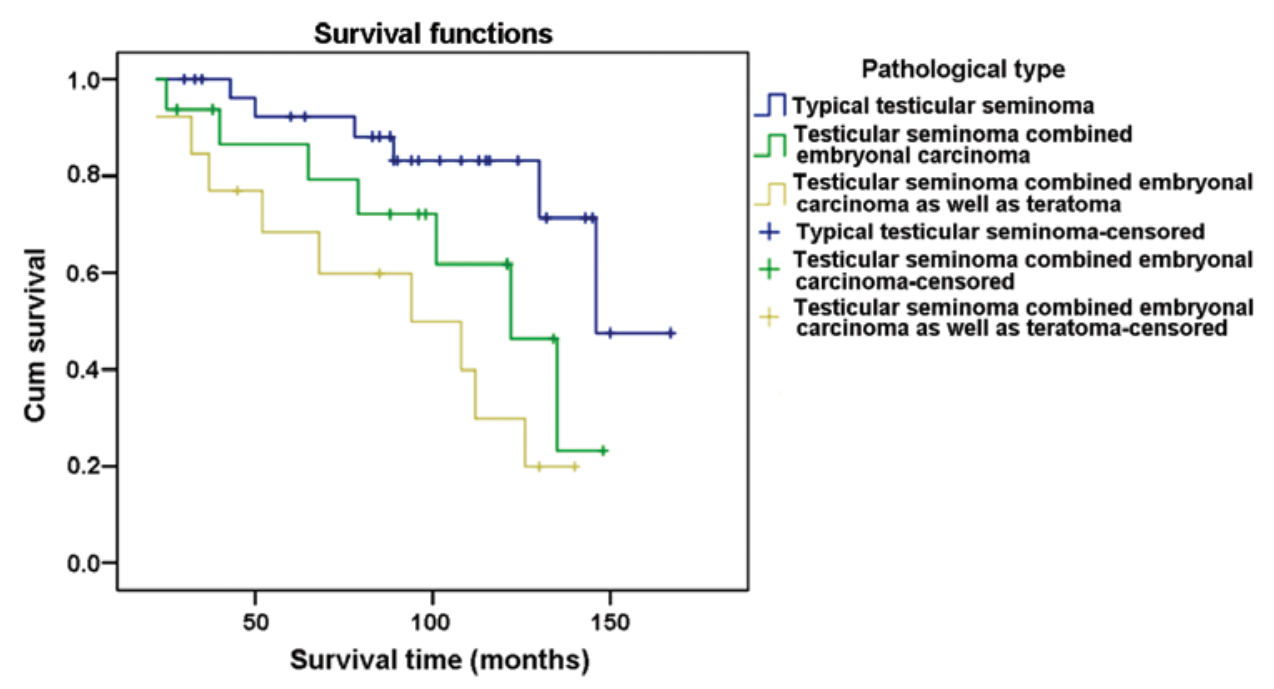

Figure 2. Comparisons on postoperative survival rate in various types of testicular seminoma.

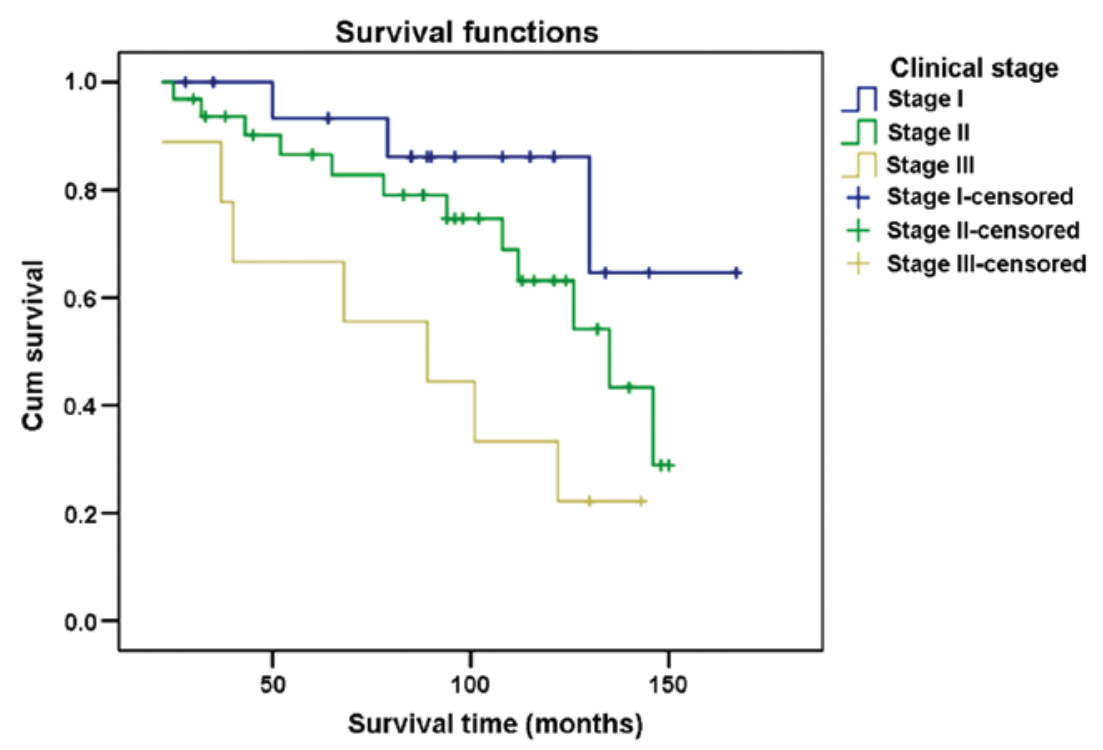

Figure 3. Effect of different stages of testicular seminoma on the prognosis. 


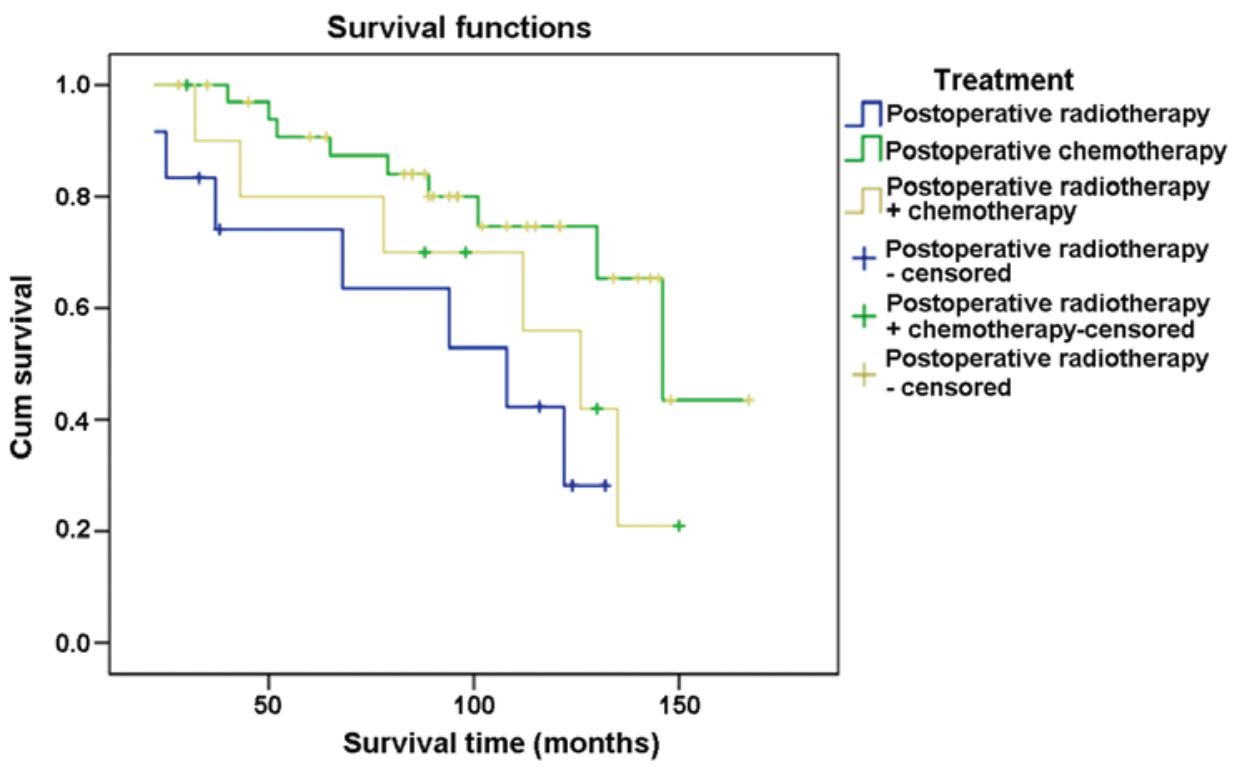

Figure 4. Survival curve for patients with testicular seminoma under different treatment methods.

Age characteristics of testicular seminoma. The peak incidence of testicular tumor was primarily evident in the $0-10$, $20-40$ and $>60$-year age groups. The incidence prior to 18 years of age was relatively small. Previous findings showed that patients aged 15-40 years had the highest incidence of testicular germ cell tumors (4). As shown in Table II, none of the enrolled 58 patients had seminoma prior to 20 years of age while the incidence for ages 30-50 was relatively high. The age distribution characteristic thus may reflect the fact that testicular seminoma is associated with sex hormones. Acquired characteristica (external factor of testis) also play a major role in the occurrence of testicular seminoma (5). Since the activation time of these factors is relatively long, the onset of testicular seminoma occurs at a late stage. Song and Huang (6) reported that of 55 testicular tumor children included in their study, aged 2-12 years, there was only one case with testicular seminoma, indicating that the onset of testicular seminoma occurs at an advanced stage.

Characteristics of the side of testicular seminoma. Current literature emphasizes that testicular germ cell tumors often occur on the right side. However, recent assessments suggested that there is no difference between the left and right sides (6). In the present study testicular germ cell tumors in $60.3 \%$ of patients (35 cases) were on the right side while in $39.7 \%$ ( 23 cases) the tumors were on the left side. The results showed that the incidence of right-side tumors was higher than that of the left-side tumors. Nonetheless, since there was a limited number of samples in the present study, whether right-side tumors had a higher frequency than those on the left side remains to be ascertained. A study with a larger sample group is likely to yield more accurate epidemiological and etiological results to determine and confirm whether the incidence of testicular seminoma is associated the position of the tumor.

Association between clinical stage, pathological types and the prognosis of testicular seminoma. Clinical stages of tumors were crucial in the selection of therapy and in forming an accurate judgment concerning prognosis. Shu et al (7) performed a multiple factor analysis on 110 cases of testicular germ cell tumors and suggested that the clinical stage of tumor was a major factor affecting the prognosis of seminoma (7). In the present study, the 3-year survival rate of clinical stages I, II and III was 100, 93.8 and $88.9 \%$, respectively; the 5-year survival rate was $94.1,87.5$ and $66.7 \%$, respectively; and the 10 -year survival rate was $88.2,71.9$ and $33.3 \%$, respectively. Differences on the 3-, 5- and 10-year survival rates of the different stages were statistically significant $(\mathrm{P}<0.05)$, which indicated that the survival rate was closely associated with clinical stage. Therefore, early identification, diagnosis and proper treatment are of great significance for the prognosis of seminoma.

The prognosis of seminomas of different pathological types varied. The 3-year survival rate of patients with typical testicular seminoma, testicular seminoma combined embryonal carcinoma, testicular seminoma combined embryonal carcinoma and teratoma was $100,93.8$ and $84.6 \%$, respectively; the 5-year survival rate was $93.1,87.5$ and $69.2 \%$, respectively; and the 10-year survival rate was $86.2,68.8$ and $38.5 \%$, respectively. The results of the log-rank single factor analysis showed that differences on the survival rate of different pathological types were statistically significant $(\mathrm{P}<0.05)$.

Serum tumor markers. Currently, LDH, AFP, HCG are the most commonly used serum tumor markers for identifying testicular germ cell tumors. Patients $(51 \%)$ with this type of cancer had increased levels of these markers (7). LDH is a marker for tissue destruction and its concentration is positively associated with tumor size. A significant increase in the LDH level is an indicative factor of a large tumor (7). On the other hand, LDH is widely identified in various tissues and its specificity is low (7). Thus, a therapy regimen cannot be determined solely on high LDH levels.

AFP and HCG are significant in determining the characteristic of testicular mass prior to surgery. They are also reliable in assessing the curative efficacy subsequent to surgery. AFP 
of seminoma was usually within the normal limit. An increase in AFP level indicates that seminoma contained mixed components, such as embryonic carcinoma (7). Higher AFP levels are associated with poorer prognosis. Nonetheless AFP is not a specific tumor marker of testicular germ cell tumors. In other malignant tumors, such as liver and gastric cancer an increase in AFP levels has been observed (7). The increasing level of HCG is associated with tumor size and prognosis. Almost $10-30 \%$ of seminoma cases containing syncytiotrophoblast had high HCG levels (7). An increase in HCG indicates that poor prognosis. However, the results of the single-factor analysis in the present study indicated that HCG level variations did not affect the prognosis of testicular seminoma $(\mathrm{P}=0.055)$ while LDH and AFP levels were statistically significant with regard to prognosis $(\mathrm{P}=0.022 ; \mathrm{P}=0.029)$.

Postoperative treatment method and prognosis. Radiotherapy has been the standard therapy for the treatment of seminoma in stages I, IIA and IIB. Postoperative adjuvant radiotherapy usually reduces the risk of local recurrence (8). However, in recent years, the importance of radiotherapy has been challenged. Zhang et al (9) reported that systemic chemotherapy was a safe and effective method for treating stage I seminoma patients following radical orchiectomy. However, other investigators have suggested radiotherapy increased the risk of second primary tumors, and did not recommend use of routine radiotherapy on patients in stage $I$ (9).

Pure abdominal radiotherapy applied on testicular seminoma in stages IIA and IIB may result in recurrence (10). Other studies revealed that in primary treatment, chemotherapy or radiotherapy combined with chemotherapy may produce improved curative effects than pure radiotherapy for patients in stages IIA and IIB (10). Patterson and associates (10) performed chemotherapy for 4-6 weeks followed by radiotherapy on their study group and reported that the 5-year survival rate without recurrence of patients in stages IIA and IIB was significantly higher than that of pure radiotherapy (11). In recent years, chemotherapy has been widely used as the first choice of treatment for patients in stages IIC and III of testicular seminoma, while chemotherapy combined with local radiotherapy is the second choice. Results from a previous study (12) revealed that the 5-year survival rate of postoperative radiotherapy, chemotherapy, and radiotherapy combined with chemotherapy was $100,91.7$ and $92.9 \%$, respectively. Results from the current study showed that the 5-year survival rate of the three classes of therapy was $91.4,75.0$ and $81.8 \%$, respectively. Notably, the differences were statistically significant.

We conclude that the 3-, 5- and 10-year survival rate of patients with testicular seminoma following orchiectomy was closely associated with the clinical stage, pathological type and postoperative adjunctive therapy. For candidates of orchiectomy, the clinical stage of the tumor must be assessed first and surgery must be performed as early as possible. Subsequently, the pathological types should be confirmed to determine the postoperative adjunctive treatment method and improve the postoperative survival rate.

\section{References}

1. Chung P, Daugaard G, Tyldesley S, Atenafu EG and Panzarella T: Evaluation of a prognostic model for risk of relapse in stage I seminoma surveillance. J Cancer Med 4: 155-160, 2015 (In Chinese).

2. Travis LB, Beard C, Allan JM, Dahl AA, Feldman DR, Oldenburg J, Daugaard G, Kelly JL, Dolan ME, Hannigan R, et al: Testicular cancer survivorship: research strategies and recommendations. J Natl Cancer Inst 102: 1114-1130, 2010.

3. Liu Fu J, Lin H and Gong X: Clinical pathology and diagnostic analysis on 15 cases of infantile testicular germ cell tumors. Natl J Androl 19: 90-93, 2013 (In Chinese).

4. Shanmugalingam T, Soultati A, Chowdhury S, Rudman S and Van Hemelrijck M: Global incidence and outcome of testicular cancer. Clin Epidemiol 5: 417-427, 2013.

5. Zheng LW, Li FB, Liu RZ, Ji RG and Zhao ZW: Clinical analysis of 87 cases of testicular tumor. Zhonghua Nan Ke Xue 11: 445-447, 2005 (In Chinese).

6. Song $\mathrm{H}$ and Huang C: Clinical analysis on children testicular tumor (attached 55 case reports). Chin J Urol 25: 44-46, 2004 (In Chinese).

7. Shu B, Liu D, Huxila D, Du X and Chen P: Multiple factor analysis on the prognosis of 110 cases with testicular germ cell tumors. J Clin Urol 26: 528-531, 2011 (In Chinese).

8. Ugwumba FO and Aghaji AE: Testicular cancer: Management challenges in an African developing country. S Afr Med J 100: 452-455, 2010.

9. Zhang X, Liu Z, Zhou F, Han H, Qin Z and Ye Y: A summary of 10 -year experience in treating stage I testicular seminoma. Cancer 29: 98-101, 2010.

10. Fucheng L: Clinical study on stage II testicular seminoma. J Contemp Urol Reprod Oncol 1: 279-281, 2009 (In Chinese)

11. Mead GM, Fossa SD, Oliver RT, Joffe JK, Huddart RA, Roberts JT, Pollock P, Gabe R and Stenning SP; MRC/EORTC seminoma trial collaborators: Randomized trials in 2466 patients with stage I seminoma: patterns of relapse and follow-up. J Natl Cancer Inst 103: 241-249, 2011.

12. Yang $\mathrm{S}$ and Wang C: Anlysis on the survival rate after testicle partial excision for 1,3,5 years of patients with testicular germ cell tumors. Chin J Hum Sex 23: 52-54, 2014 (In Chinese). 